Erschienen in: Augst, Gerhard/Blüml, Karl/Nerius, Dieter/Sitta, Horst (Hrsg.):

Zur Neuregelung der deutschen Orthographie. Begründung und Kritik.

Tübingen: Niemeyer, 1997. S. 365-378. (Reihe Germanistische Linguistik 179)

Dieter Herberg

\title{
5.3 Aussageabsicht als Schreibungskriterium - ein alternatives Reformkonzept für die Regelung der Getrennt- und Zusammenschreibung (GZS)
}

Bei den Beratungen des Internationalen Arbeitskreises für Orthographie über die Neuregelung der deutschen Rechtschreibung lagen in bezug auf die GZS zwei Reformvorschläge auf dem Tisch. Neben dem Vorschlag der IDS-Kommission für Rechtschreibfragen, der dann als Grundlage für die später beschlossene Reformregelung diente (vgl. dazu 3.4 Schaeder in diesem Band), lag der Vorschlag der DDR-Forschungsgruppe Orthographie, der das gemeinsame Ziel einer Vereinfachung der bisherigen Regelung mit einem zum Teil anderen Ansatz zu erreichen versuchte. Da dieser Reformvorschlag, der von mir im Rahmen der Forschungsgruppe Orthographie ausgearbeitet worden ist (vgl. Herberg 1981, 1986), in seiner endgültigen Fassung, die den Teilnehmern der Züricher Tagung vom September 1987 vorlag, noch nicht veröffentlicht worden ist, nehme ich gern die Gelegenheit wahr, im Rahmen dieser Publikation auf ihn zurückzukommen.

Nach einem kurzen Rückblick auf die Behandlung der GZS-Problematik im Internationalen Arbeitskreis (1.) gehe ich auf die Funktion der GZS in der deutschen Gegenwartssprache ein (2.), leite daraus die Grundsätze für den Neuregelungsvorschlag ab (3.), gebe den Vorschlag im Wortlaut vollständig wieder (4.) und gehe abschließend auf die wesentlichen Gemeinsamkeiten und Unterschiede dieses Vorschlages in bezug auf die beschlossene Neuregelung ein (5.).

\section{Die GZS-Problematik im Internationalen Arbeitskreis für Orthographie}

Im Rahmen der Arbeitstagungen, auf denen der Internationale Arbeitskreis für Orthographie zwischen 1980 und 1991 systematisch Probleme der deutschen Rechtschreibung und Vorschläge für deren Neuregelung erörterte, stand der orthographische Teilbereich der Getrennt- und Zusammenschreibung (GZS) - gemeinsam mit anderen Problemen - bei den Tagungen von 1986 (Mannheim), von 1987 (Zürich) und von 1988 (Rostock) auf der Tagesordnung.

Nachdem auf der Mannheimer Arbeitstagung von 1986 zunächst «von Dieter Herberg (Forschungsgruppe DDR) und Burkhard Schaeder (Kommission IDS) in zwei Grundsatzreferaten Grundfragen der Zusammen- und Getrenntschreibung behandelt» worden waren, verständigten sich die Teilnehmer «über die Grundsätze der Ausarbeitung eines neuen Regelvorschlags für die Zusammen- und Getrenntschreibung und stimmten überein, daß dafür die entsprechenden Vorarbeiten in den einzelnen Arbeitsgruppen durchzuführen sind» (Mentrup 1986: 286). Auf der Arbeitstagung von 1987 in Zürich war die GZS dann der Hauptberatungsgegenstand. Der Chronist berichtet: «Nach der Bestimmung der z. T. recht konträren Standorte der einzelnen Arbeitsgruppen (Dieter Herberg, Burkhard Schaeder, Ernst Holzfeind, Horst Sitta) wurde auf der Grundlage des Regelvorschlages der Forschungsgruppe Orthographie der DDR (Berlin/Rostock) und der 
Kommission für Rechtschreibfragen des IDS (Mannheim) mit der Erarbeitung neuer Regeln begonnen» (Mentrup 1987: 380). Das mühselige Geschäft, den in Zürich entwickelten Vorschlag im Detail auszuführen, wurde Burkhard Schaeder und Dieter Herberg übertragen (vgl. dazu im einzelnen 3.4 Schaeder diesem Band); als Ergebnis lag auf der Rostocker Arbeitstagung 1988 zur «Beratung und Beschlußfassung [...] ein - auf der Basis der Verhandlungen der letzten internationalen Konferenz - von Dieter Herberg und Burkhard Schaeder gemeinsam erarbeitetes (Regelwerk zur Neuregelung der Getrennt- und Zusammenschreibung) vor» (Schaeder 1989: 89). Mit Recht weist Schaeder auf den folgenden Umstand hin: «Die schließlich endgültig verabschiedete, vorher bereits international abgestimmte Fassung entspricht - von einigen Regelformulierungen, Regelerweiterungen und Regelumstellungen einmal abgesehen - insgesamt jener, die im 〈Vorschlag zur Neuregelung der deutschen Rechtschreibung) der Kommission für Rechtschreibfragen des Instituts für deutsche Sprache (1989) präsentiert wird» (1989: 90). In der Tat hatte sich in Zürich die Mehrheit der Teilnehmer dafür ausgesprochen, der Weiterarbeit am Regelwerk den IDS-Vorschlag zugrunde zu legen. Zwar wurden einzelne Elemente auch aus dem DDR-Vorschlag berücksichtigt (z. B. die Bezeichnung dieses Bereiches als «Getrennt- und Zusammenschreibung», die Herauslösung der Bindestrich-Schreibung als eigenständiger Teilbereich), aber insgesamt spielte dieser Reformvorschlag im Internationalen Arbeitskreis nach der Grundsatzentscheidung für den IDS-Vorschlag keine Rolle mehr.

\section{Zur Funktion der GZS in der deutschen Gegenwartssprache'}

Betrachtet man zunächst die Funktion der Schreibung bei Buchstabenschriften generell, so besteht sie zum einen in der mehr oder weniger unmittelbaren Wiedergabe der Lautung, um so die flüchtige gesprochene Sprache bewahrbar und wiederholbar zu machen. Diese Teilfunktion, die wir als Aufzeichnungsfunktion der Schreibung bezeichnen, dominierte ursprünglich. Mit der Entwicklung der geschriebenen Sprache und der allgemeinen Verbreitung des stillen Lesens hat sich die Funktion der Schreibung erweitert; mit ihrer Hilfe werden nun auch mehr und mehr semantische Informationen direkt und ohne Vermittlung über die Lautung zum Ausdruck gebracht. Bestimmte semantische Beziehungen, Zusammenhänge und Unterschiede werden unmittelbar verdeutlicht, um so die Erfassung des Inhalts geschriebener Texte beim Lesen zu unterstützen. Diese Teilfunktion der Schreibung bezeichnen wir als Erfassungsfunktion (vgl. zu den Funktionen der Schreibung Nerius et al. 1989: 22ff.). Beide Funktionen entsprechen legitimen Anforderungen der Sprachbenutzer, und nur ihr ausgewogenes Zusammenspiel sichert das störungsfreie Funktionieren der Kommunikation mittels geschriebener Sprache.

In bezug auf die GZS heißt das: Die getreue Abbildung des Lautkontinuums der gesprochenen Sprache - gemäß der Aufzeichnungsfunktion der Schreibung wird im Laufe der historischen Entwicklung allmählich zurückgedrängt, weil sich

1 Die Darlegungen in diesem Abschnitt folgen im wesentlichen meiner gedrängten Darstellung in Herberg (1988). 
die graphische Markierung von semantischen Einheiten für den Lesenden im Interesse der besseren und schnelleren Sinnerfassung als wesentlicher erwies. Wir erkennen also in der Gliederung von geschriebenen Äußerungen in graphische Wortformen, unter denen wir Graphemfolgen zwischen zwei Leerstellen (Spatien) verstehen, ein spezielles Verfahren der Schreibung zur Erfüllung ihrer Erfassungsfunktion. Demselben Ziel wie die primäre Gliederung geschriebener Äußerungen in graphische Wortformen, nämlich zur Unterstützung der Sinnentnahme Bedeutungseinheiten formal zu kennzeichnen, dient auch die - nur scheinbar entgegengesetzt gerichtete - sekundäre graphische Zusammenziehung bestimmter Typen von semantisch-syntaktisch aufeinander bezogenen Wörtern, von der das Deutsche bei der Wortbildung reichen Gebrauch macht. Im Laufe der sprachlichen Entwicklung wurde der Verzicht auf das Spatium zwischen (zwei) Graphemfolgen der genannten Art immer bewußter und in immer größerem Umfang als Mittel für die Weitergabe spezieller Informationen an den Lesenden genutzt, was zur Kompliziertheit der GZS-Regelung beigetragen hat.

Die heutige Hauptfunktion der GZS besteht darin, den Lesenden darüber zu informieren, in welche Weise er die semantisch-syntaktischen Beziehungen «nebeneinanderstehender gedanklich zusammengehörige Wörter» - so die Formulierung im Leipziger Duden (1985), auf den wir uns hier und im folgenden beziehen - interpretieren soll: als syntaktisch selbständige und semantisch eigenständige Glieder einer Wortgruppe oder als unmittelbare Konstituenten einer Wortbildungskonstruktion in Gestalt eines zusammengesetzten Wortes, eines Kompositums.

Die Getrenntschreibung (GS) oder die Zusammenschreibung (ZS) können dem Lesenden somit Hinweise auf bestimmte Aussageabsichten des Schreibenden geben und fungieren gleichsam als Lese- und Verständnishilfe. In neuerer Zeit - besonders mit zunehmender Kodifizierung - wurden die GS und die ZS über die Markierung des kategorialen Unterschiedes Wortgruppe vs. Kompositum hinaus mit semantischen und syntaktischen Informationen verknüpft, die zum Teil nicht aus diesem Grundunterschied erklärbar sind, mitunter auch im Widerspruch dazu stehen (z. B. konkreter vs. übertragener Gebrauch; attributive vs. prädikative Verwendung). Die Probleme der GZS ergeben sich also - wenn man es so ausdrücken will - im Berührungs- und Überschneidungsbereich von Einheiten der syntaktischen Ebene (Wortgruppe) und der lexikalischen Ebene (Wort) des Sprachsystems.

Das hat dazu geführt, daß die GZS herkömmlicherweise unterschiedlichen, zum Teil auch mehreren der traditionellen sogenannten orthographischen oder Schreibungsprinzipien zugeordnet wird, so dem «grammatischen», dem «logischen», dem «semantischen» Prinzip (vgl. zum Begriff des orthographischen Prinzips Nerius et al. 1989: 68ff.). Im Unterschied dazu vertreten wir die Auffassung, da $B$ jede orthographische Erscheinung dem Prinzip zugeordnet werden sollte, das diejenigen Beziehungen zwischen einer der anderen Ebenen des Sprachsystems zu dessen graphischer Ebene ausdrückt, die für die gegebene orthographische Erscheinung im geschriebenen Gegenwartsdeutsch prägend sind. Das ist im Falle der GZS das lexikalische Prinzip (vgl. dazu Herberg 1980). Seine Dominanz in diesem orthographischen Bereich zeigt sich vor allem darin, daß im Deutschen seit längerer Zeit die Tendenz herrscht, bei bestimmten Typen von Wortgruppen, 
die einen relativ abgegrenzten, einheitlichen Sachverhalt der Realität (Gegenstand, Eigenschaft, Vorgang, Beziehung) benennen und damit eine der der Einwortlexeme ähnliche Benennungsfunktion übernehmen, auch die geschriebenen Formative denen von Einwortlexemen anzugleichen und sie in eine geschlossene orthographische Wortform zu überführen (graphische Univerbierung). Daneben wird die GZS vom syntaktischen und vom intonatorischen Prinzip beeinflußt.

Die Beobachtung, daß die Formseite sprachlicher Einheiten im Laufe der Entwicklung hinter der inhaltlichen Seite zurückbleibt, gilt in besonders hohem Maße für die einmal kodifizierte graphische Form. So gibt es zu jeder Zeit und natürlich auch gegenwärtig und künftig bei bestimmten Typen von Kombinationen eine Übergangszone von Fällen, die zwar noch die graphische Form von Wortgruppen, aber schon inhaltliche Merkmale eines Kompositums haben, z. B. instand setzen, warm machen; nach Hause, statt dessen; mit Hilfe. Der Umstand, $\mathrm{da} ß$ es sich hierbei um einen fortwährenden Entwicklungsprozeß handelt, dem die Kodifizierung der Schreibungsnormen aufgrund ihres statischen Charakters nur ungenügend gerecht werden kann, ist der Grund für zahlreiche orthographische Zweifelsfälle. Zudem hat die Uneinheitlichkeit des Gegenstandbereiches und haben Einflüsse der Kodifizierungsgeschichte zu einer schwer überschaubaren und funktional überbelasteten Regelung der GZS geführt, die Überlegungen zu ihrer Vereinfachung herausfordert.

\section{3. $\mathrm{Zu}$ den Grundsätzen der hier vorgeschlagenen Neuregelung der GZS}

Entsprechend den theoretischen Grundpositionen der Forschungsgruppe Orthographie (vgl. Nerius et al. 1989), in deren Rahmen auch der unter 4. wiedergegebene Vorschlag für eine Neuregelung der GZS entwickelt wurde, ist es das Ziel eines jeden reformerischen Eingriffs in das Gefüge der Orthographie, die Schreibung auf dem betreffenden Teilgebiet zu optimieren und damit zur optimalen Erfüllung der Funktion der geschriebenen Sprache überhaupt beizutragen. Die Schreibung ist um so günstiger gestaltet, je konfliktfreier sie ihren beiden Funktionen, d. h. ihrer Aufzeichnungsfunktion und ihrer Erfassungsfunktion, gerecht zu werden vermag, oder anders gesagt: je besser sie die legitimen Belange sowohl des Schreibenden (also größtmögliche Einfachheit im Interesse der leichten Erlernbarkeit und Handhabung) als auch des Lesenden (also direkte Bedeutungsverdeutlichung und leichte Überschaubarkeit im Interesse der raschen Informationsentnahme) befriedigt.

Die Analyse der gegenwärtig geltenden GZS-Regelung hatte ergeben, daß die Proportionen zwischen den beiden funktionsbezogenen Anforderungen an die Schreibung zum Nachteil einer einfachen Regelung verschoben sind (vgl. Herberg 1975, 1981, 1986). Da die Legitimität beider Anforderungen grundsätzlich anerkannt wird, kann ein Vereinfachungsvorschlag z. B. nicht den radikalen Abbau der Schreibungen in Betracht ziehen, die die direkte Informationsentnahme unterstützen, um auf diese Weise den Grundsatz der Einfachheit durchzusetzen. Vielmehr muß erwogen werden, ob entweder durch konsequentere Anwendung von in der geltenden Regelung angelegten Grundsätzen eine klare und befriedigende Lösung zu erreichen ist oder ob etwa die Freigabe der Schreibung in diesem Bereich das angestrebte Gleichgewicht herstellen würde. 
Die Neuregelung sollte für den Schreibenden auf jeden Fall eine Verringerung des Aufwandes an Zeit und Mühe bei der Erlernung der GZS und bei ihrer Handhabung bewirken, ohne da $\beta$ dadurch für den Lesenden Nachteile bei der raschen Sinnerfassung des Textes entstehen. Im Gegenteil: Der Informationswert der GZS soll durch ihre funktionale Entlastung eindeutig und damit kommunikativ wirksamer gemacht werden. Diesem Ziel versucht unser Neuregelungsvorschlag auf folgende Weise zu entsprechen:

Der Signalwert des Spatiums bzw. der seiner Abwesenheit zwischen aufeinander bezogenen benachbarten Graphemfolgen soll prinzipiell auch künftig genutzt werden und unterliegt somit der Notwendigkeit der Regelung. Die Regelung muB deutlich machen, daß es sich bei der GZS um den graphisch-formalen Reflex semantischer Gegebenheiten der syntaktischen und der lexikalischen Ebene handelt (Wortgruppe oder zusammengesetztes Wort). Auf der Basis der kategorialen Spezifik der Wortarten, denen die benachbarten Bestandteile angehören, müssen die Regeln für jeden der von der GZS-Problematik betroffenen Bereiche, also für den verbalen, den adjektivischen, den partizipialen und den Bereich der Adverbien, Präpositionen und Konjunktionen das jeweils wesentliche semantische (kategoriale) Kriterium enthalten, das im Bereich der betreffenden Wortarten den Inhalt der Wortgruppe bzw. der Zusammensetzung ausmacht. Die entsprechende Schreibung folgt als graphische Realisationsform mit Zwangsläufigkeit: GS für Wortgruppen, ZS für Komposita.

Informationen, die über den semantisch-kategorialen Unterschied zwischen Wortgruppe und Kompositum hinausgehen und diesen überlagern oder verunklaren, sollen von der GZS nicht mehr transportiert werden. Das bedeutet vor allem, $\mathrm{da} ß$ auf die graphische Markierung von metaphorischem (übertragenem) Gebrauch durch ZS gegenüber konkretem Gebrauch mit GS verzichtet wird. Im übrigen ist es mit unserer Auffassung von der Bilateralität sprachlicher Zeichen und von der Einheit des Wortes nicht vereinbar, daß Sememe ein und desselben Zeichens - und die metaphorische Übertragung ist eine Beziehung zwischen Sememen - einmal einem Wortformativ (kaltstellen), einmal einem Wortgruppenformativ (kalt stellen) als Zeichenkörper zugeordnet sein sollen. Der graphische Ausgleich kann in der Weise erfolgen, daß die betreffenden Wortgruppen in eine graphische Wortform überführt werden, womit die Einheit des Wortes in diesen Fällen hergestellt ist. Dieser Lösungsweg, der besonders in Teilen des verbalen Bereiches zu in gewissem Umfang vermehrter Zusammenschreibung führt, befindet sich in Übereinstimmung mit der beobachteten Univerbierungstendenz.

$\mathrm{Zu}$ der prinzipiellen Unterscheidung von Wortgruppe (mit GS) und Kompositum (mit ZS) gehört, daß sie unabhängig von der attributiven oder prädikativen Verwendung im Satz gilt und dieser übergeordnet ist. Die im adjektivischen und partizipialen Teilbereich bisher üblichen, aber wenig verbindlichen Schreibungsempfehlungen mit der Differenzierungstendenz attributiv $=\mathrm{ZS} /$ prädikativ $=$ GS (leichtverdauliche Speisen / die Speisen sind leicht verdaulich) werden nicht aufrecht erhalten, da auch sie die GZS-Regelung belasten, deren eigentliche Funktion verdunkeln und kommunikativ kaum wirksam sind.

In diesen Orientierungen und Grundsätzen sehen wir den Rahmen für einen gangbaren Weg zu einer GZS-Regelung, die gekennzeichnet ist durch geringe Regelzahl aufgrund funktionaler Entlastung, schlüssige Kriterien und wenig 
Ausnahmen und die damit geeignet ist, in diesem Bereich der Schreibung zur optimalen Erfüllung der Funktion von geschriebener Sprache beizutragen.

Der im folgenden im Wortlaut wiedergegebene Reformvorschlag versucht, einige wesentliche Nachteile sowohl der jetzt geltenden Regelung als auch anderer Reformvorschläge zu vermeiden, ohne den Anspruch zu erheben, der Weisheit letzter Schluß zu sein. Der Vorschlag kann knapp folgendermaßen gekennzeichnet werden: Er knüpft an die geltende Regelung an, entwickelt sie in Richtung auf wirksamere Funktionserfüllung weiter und befindet sich in Übereinstimmung mit Entwicklungstendenzen der deutschen Standardsprache. Er macht die Aussageabsicht des sprachbewußt Schreibenden zum obersten Gesichtspunkt der Regelung, und er nutzt durch funktionale Entlastung den Signalwert der GZS für die Bedeutungserfassung wirksamer aus, indem er ihn konsequent aus dem Grundunterschied zwischen Wortgruppe und Zusammensetzung ableitet.

Die Erlernung und Handhabung der vorgeschlagenen Regelung werden durch die erhebliche Reduzierung der Regelzahl, durch ihre durchsichtige Gestaltung und durch die Beschränkung von Schreibungsänderungen auf ein unumgängliches Mindestmaß unterstützt. Insgesamt bleiben bei deutlich absehbaren Erleichterungen für die Aneignung und Handhabung dieser GZS-Regelung die Normänderungen im lexikalischen System (Wörterverzeichnis) und deren Auswirkungen auf die Textgestalt so maßvoll, daß im Reformfall auf diesem Teilgebiet keine gravierenden Umstellungs- und Anpassungsschwierigkeiten zu erwarten wären (vgl. Herberg 1986: 284ff.). Damit entspricht unser GZS-Neuregelungsvorschlag der von der Forschungsgruppe Orthographie verfolgten gemäßigten Reformlinie.

\section{Der Vorschlag}

Vorschlag zur Regelung der Getrennt- und Zusammenschreibung

\section{Vorbemerkungen}

Regeln

I Verb

I.1 Substantiv + Verb (R 1, R 2)

I.2 Adjektiv + Verb (R 3, R 4)

I.3 Verb + Verb (R 5, R 6)

I.4 Adverb / Präposition + Verb (R 7, R 8, R 9)

II Adjektiv / Partizip

II. I Substantiv / Verbalstamm / Pronomen / Adverb / Präposition / Adjektiv / Partizip + Adjektiv (R 10, R 11, R 12)

II.2 Adjektiv / Adverb / Pronomen + Partizip (R 13, R 14)

II.3 Substantiv + Partizip (R 15, R 16)

III Adverb / Präposition / Pronomen, Konjunktion, Numerale (R 17, R 18, R 19, R 20)

IV Substantiv (R 21) 


\section{Vorbemerkungen}

1. Die Getrennt- und Zusammenschreibung betrifft die Schreibung von Wörtern, die im Text benachbart und aufeinander bezogen sind: Getrennt werden die Bestandteile von Wortgruppen geschrieben; zusammen werden die Bestandteile von Zusammensetzungen (Komposita) geschrieben.

2. Manchmal können dieselben Bestandteile sowohl eine Wortgruppe als auch eine Zusammensetzung bilden. Die Verwendung als Wortgruppe oder als Zusammensetzung hängt dann oft von der Aussageabsicht des Schreibenden ab.

Bei verschiedenen Wortarten sind dabei - auch in Abhängigkeit von in Gang befindlichen sprachlichen Entwicklungsprozessen - spezielle Bedingungen zu beachten. Daher ist die Regelung nach Wortarten gegliedert.

3. In den Regeln werden folgende Symbole verwendet:

$$
\begin{aligned}
& \mathrm{R}=\text { Regel } \\
& \mathrm{B}=\text { Beispiel } \\
& \mathrm{E}=\text { Erläuterung }
\end{aligned}
$$

\section{Regeln}

I Verb

Nur beim Verb stehen der Wortgruppe (z. B. Verdacht schöpfen) zwei Arten der Zusammensetzung gegenüber: Die typische trennbare Zusammensetzung, die in den infiniten Formen und den finiten Formen bei Endstellung zusammen-, in den finiten Formen bei Erst- und Zweitstellung aber getrennt geschrieben wird (z. B. radfahren, radfahrend, radgefahren, um radzufahren; ich fahre rad, fährt er rad?), und die untrennbare Zusammensetzung, die in allen Formen zusammengeschrieben wird (z. B. schlußfolgern, schlußfolgernd, geschlußfolgert, um zu schlußfolgern, ich schlußfolgere, was schlußfolgerst du?).

Wenn nicht ausdrücklich etwas anderes gesagt ist, handelt es sich stets um trennbare Zusammensetzungen.

\section{I.1 Substantiv + Verb}

R I Das Substantiv wird vom Verb getrennt geschrieben, wenn es als Akkusativobjekt trotz fehlender formaler Kennzeichnung (durch Präposition oder Artikel) gegenüber dem Verb selbständig und inhaltlich dominierend ist.

B: Faxen machen, Feuer fangen, Versteck spielen, Walzer tanzen; Bescheid geben, Ernst machen, Folge leisten, Fuß fassen, Gefahr laufen, Gefallen finden, Ränke schmieden, Rechnung tragen, Schaden nehmen, Verdacht schöpfen

R 2 Das Substantiv wird mit dem Verb zusammengeschrieben, wenn es als Bestimmungswort den Vorgang näher charakterisiert:

B: ballspielen: ich spiele ball, ich habe ballgespielt, um ballzuspielen, ballspielend.

bockspringen, eistanzen, maschineschreiben, postenstehen, radfahren, schlangestehen, schlittenfahren, skilaufen; gewährleisten, maßhalten, preisgeben, rechtsprechen, schritthalten, teilhaben 
E 2 Seltener bilden Substantiv und Verb untrennbare Zusammensetzungen; oftmals sind von ihnen nicht sämtliche Formen, sondern vor allem Infinitiv und Partizip II gebräuchlich.

B: schlußfolgern, lobpreisen, nachtwandeln, nasführen;

bergsteigen: ich bin berggestiegen

bauchreden, bruchlanden, bruchrechnen, brustschwimmen, kopfrechnen, notlanden, punktschweißen, schutzimpfen, segelfliegen, seilspringen, strafversetzen, wettrennen, zwangsräumen

\section{I.2 Adjektiv + Verb}

R 3 Das Adjektiv wird vom Verb getrennt geschrieben, wenn es den Vorgang qualitativ charakterisiert (Frage: wie?). Beide Bestandteile der Wortgruppe tragen bei neutraler Aussprache einen Akzent.

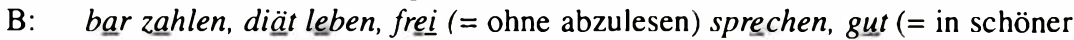
Schrift, in gutem Stil) schrêiben, heimlich (= im geheimen) tün, laut reden. richtig (= ordnungsgemäß) stellen, sicher (= ohne Beeinträchtigung) gehen

R 4 Das Adjektiv wird mit dem Verb zusammengeschrieben, wenn es das Resultat des Vorgangs nennt.

Der Akzent liegt bei neutraler Aussprache auf dem adjektivischen Bestandteil der Zusammensetzung.

B: bekanntgeben, dichtmachen, freisprechen (von Schuld), gütschreiben (einen Betrag), heimlichtun (= geheimnisvoll tun), irreführen, kaltstellen (= kühlen; einflußlos machen), lautwerden [lassen], nahegehen, richtigstellen (= berichtigen), sichergehen (gewiß sein), wahrmachen

E 4 Zusammengeschrieben wird auch, wenn sich das Adjektiv zum reihenbildenden Präfixoid entwickelt hat oder als selbständiges Wort in dieser Bedeutung nicht mehr vorkommt.

B: fehlgehen, -leiten, -orientieren, -schlagen; loslegen, -schlagen, -werden; schwarzarbeiten, -fahren, -hören, -sehen; i $r$ refiuhren, weismachen

I.3 Verb + Verb

R 5 Verben werden voneinander getrennt geschrieben (vgl. aber R 6).

$\mathrm{B}$ : baden gehen, bleiben lassen, gehen müssen, haften bleiben, kommen sehen, lachen machen, lesen lernen, schlafen gehen, spazieren fahren, stecken bleiben, warten können, waschen helfen

R 6 Zwei Verben werden zusammengeschrieben, wenn die Bedeutung des zweiten Verbs stark verblaßt ist. Es handelt sich um wenige Fälle mit -gehen und -lernen.

B: flötengehen, verlorengehen; kennenlernen, liebenlernen, schätzenlernen

I.4 Adverb / Präposition + Verb

R 7 Das Adverb bzw. die Präposition werden vom Verb getrennt geschrieben, wenn sie als Adverbialbestimmung oder als Teil des Prädikats (Prädikativ) selbständiges Satzglied sind. 
B: Wir wollen aneinander denken; ihr könnt da bleiben, wo ihr jetzt seid; sie soll um 5 Uhr da (= an der bezeichneten Stelle) sein; so etwas mußt du künftig vorher sagen; sie werden den Preis wieder (= nochmals) gewinnen; wir wollen den Korb zusammen (= gemeinsam) tragen;

die Treppe hinauf rennen (nicht: gehen);

auf und $a b$ gehen (= ohne bestimmte Richtung); hin und her fahren (= kreuz und quer)

R 8 Das Adverb bzw. die Präposition werden mit dem Verb zusammengeschrieben, wenn sie dessen Bedeutung genauer bestimmen.

B: Perlen aneinanderreihen; länger kann ich nicht dableiben; wirst du dasein, wenn er kommt?; ein Ergebnis vorhersagen (= prophezeien); sie werden den Preis wiedergewinnen (= zurückgewinnen); Fakten zusammentragen (=sammeln); die Treppe hinaufrennen (nicht: hinunter-); während der Fahrt auf- und abspringen; hin- und herfahren (= hin- und wieder zurückfahren)

E 8 Abweichend von $\mathrm{R} 8$ wird getrennt geschrieben, wenn der adverbiale / präpositionale Bestandteil in finiten Formen mit der Absicht besonderer Hervorhebung umgestellt wird.

B: Nicht einmal an sah sie ihn dabei

Aber: Während sie ihn ansah...;

Aus hielt er, bis er das Ufer gewann (Fontane)

Aber:... weil er das nicht mehr aushielt;

Zusammen schreibt man, wenn ...

Aber: Obwohl man hier in der Regel zusammenschreibt...

R 9 Die Präpositionen durch, hinter, über, um, unter, wider sowie das Adverb wieder bilden mit Verben außer trennbaren auch untrennbare Zusammensetzungen. Der Akzent liegt auf dem verbalen Bestandteil.

B: durchfahren: der Bus durchfährt die Ortschaft aber: durchfahren: der Bus fährt an dieser Station durch

hinterlegen: eine Nachricht beim Pförtner hinterlegen aber: hinterlegen: vor Fahrtantritt hat sie den Mantel hintergelegt (im Auto) übersetzen: einen Artikel ins Russische übersetzen aber: $\underline{\ddot{u} b e r s e t z e n: ~ s i e ~ s e t z t e n ~ m i t ~ d e r ~ F a ̈ h r e ~ u ̈ b e r ~}$ umfahren: er hat das Hindernis geschickt umfahren aber umfahren: er hat den Kegel umgefahren unterstellen: diese Äußerung hat er ihr unterstellt aber: unterstellen: er hat seinen Wagen bei uns untergestellt widersprechen: er widerspricht mir ständig aber: widerhallen: in dem Gewölbe hallt es wider wiederholen: den Unterrichtsstoff wiederholen aber: wiederholen: einen Siegertitel wiederholen

\section{Adjektiv / Partizip}

Da viele Partizipien wie Adjektive verwendet werden, unterliegen sie auch großenteils denselben Regeln der Getrennt- und Zusammenschreibung wie die Adjektive. Auf einige Besonderheiten wird hingewiesen. 
II. 1 Substantiv / Verbalstamm / Pronomen / Adverb / Präposition / Adjektiv / Partizip + Adjektiv

R 10 Das Substantiv / das Adjektiv / der Verbalstamm / das Pronomen / das Adverb / die Präposition wird mit dem Adjektiv zusammengeschrieben.

B: butterweich, felsenfest, hilfsbedürftig, lebensfremd, meterhoch; bitterböse, hellrot, lauwarm, naßkalt, taubstumm, tollkühn; fahrtüchtig, lernbegierig; selbstgefällig; immergrün; vorlaut

R 11 Das Adjektiv / das Partizip / das Adverb nicht wird vom Adjektiv getrennt geschrieben, wenn es ein Merkmal dieses Adjektivs bezeichnen soll. Mit solchen Wortgruppen bezieht man sich meist auf einen konkreten Einzelfall (vgl. R 12).

B: eine schwer verständliche Abhandlung - diese Abhandlung ist schwer verständlich

ein leicht verdauliches Essen; eine gut bürgerliche Ausstattung; klein karierte Kissenbezüge; sein blendend weißes Hemd; ein strahlend heller Morgen; eine nicht öffentliche Beratung

R 12 Das Adjektiv / das Partizip / das Adverb nicht wird mit dem Adjektiv zusammengeschrieben, wenn es mit diesem zusammen ein Merkmal oder eine Eigenschaft von etwas bezeichnen soll. Mit so zusammengesetzten Adjektiven bezieht man sich meist auf eine ganze Klasse von Fällen (vgl. aber R 11).

B: schwerverständliche Romane - seine Romane sind schwerverständlich leichtverdauliche Gerichte; gutbürgerliche Küche; kleinkarierte Ansichten; blendendweiße Fassaden; strahlendhelle Gletscher; nichtöffentliche Verhandlungen.

II.2 Adjektiv / Adverb / Pronomen + Partizip

R 13 Das Adjektiv / das Adverb / das Pronomen wird vom Partizip getrennt geschrieben, wenn es ein Merkmal dieses Partizips bezeichnen soll. Mit solchen Wortgruppen bezieht man sich meist auf einen konkreten Einzelfall (vgl. aber R 14).

B: das reich geschmückte Haus - das Haus ist reich geschmückt eine blank polierte Klinke; wir sind eng befreundet; hast du das ernst gemeint?; ein hell strahlender Stern; ein voll besetzter Bus; der Fall wurde viel besprochen; seine nicht erfültten Wünsche; der oben erwähnte Autor; ihr selbst gebackener Kuchen

R 14 Das Adjektiv / das Adverb / das Pronomen wird mit dem Partizip zusammengeschrieben, wenn es mit diesem zusammen ein Merkmal oder eine Eigenschaft von etwas bezeichnen soll. Mit zusammengesetzten Partizipien bezieht man sich meist auf eine ganze Klasse von Fällen (vgl. aber R 13).

B: reichgeschmückte Straßen - die Straßen waren reichgeschmückt blankpolierte Schilder; engbefreundete Ehepaare; ernstgemeinte Vorschläge; hellstrahlende Gesichter; vollbesetzte Züge; ein vielversprechendes Buch; nichterfüllte Erwartungen; die obenerwähnte Situation; selbstgebackene Plätzchen 


\section{II.3 Substantiv + Partizip}

Hier gelten für Partizip I und Partizip II unterschiedliche Regeln.

R 15 Das Substantiv wird vom Partizip I entsprechend R 13 getrennt geschrieben, wenn es ein Merkmal dieses Partizips bezeichnen soll, und wird mit dem Partizip I entsprechend R 14 zusammengeschrieben, wenn es mit diesem zusammen ein Merkmal oder eine Eigenschaft von etwas bezeichnen soll.

B: $\quad$ ein Klavier spielendes Kind - alle klavierspielenden Schüler;

Aufsehen erregend - aufsehenerregend; Erfolg versprechend-erfolgversprechend; Feuer speiend - feuerspeiend; Gewinn bringend - gewinnbringend; Laub tragend - laubtragend

R 16 Das Substantiv wird mit dem Partizip II stets zusammengeschrieben, um mit diesem zusammen ein Merkmal oder eine Eigenschaft von etwas zu bezeichnen.

B: haßerfüllt, hausgemacht, notgedrungen, sonnenverbrannt, staubbedeckt, strahlengeschädigt, wahlberechtigt, wutentbrannt

E 16 Auch eine Reihe von Kombinationen aus Substantiv + Partizip I wird nur zusammengeschrieben. In den entsprechenden Wortgruppen sind die Substantive mit Artikel und/oder Präposition versehen.

B: ausschlaggebend - den Ausschlag gebend postlagernd - auf der Post lagernd

fettriefend - von Fett triefend

durstlöschend, epochemachend, freudestrahlend, herzzerreißend, postwendend, spanabhebend, stellvertretend, tonangebend

III Adverb / Präposition / Pronomen, Konjunktion, Numerale

R 17 Fügungen aus den verschiedensten Wortarten, die wie Adverbien / Präpositionen / Pronomen fungieren, werden getrennt geschrieben, wenn die Wortart, die Bedeutung und die Wortform ihrer einzelnen Bestandteile erhalten ist.

B: (wie Adverbien:) außer Atem, darüber hinaus, im besonderen, nach wie vor, vor allem, zum zweiten Mal, zu Rate, zur Zeit

(wie Präpositionen:) aus Anlaß, im Interesse, in Anbetracht, mit dem Ziel, nach Maßgabe, unter Verwendung (von), zu Lasten, zum Zweck

(wie Pronomen:) gar kein, so etwas

R 18 Aus Fügungen entstandene Adverbien / Präpositionen / Pronomen werden zusammengeschrieben, wenn die Wortart, die Bedeutung und die Wortform ihrer einzelnen Bestandteile nicht mehr deutlich oder zugunsten der neuen Wortart aufgegeben worden sind.

B: (Adverbien:) außerstande, beidemal, daraufhin, garnicht, inmitten, insbesondere, vielerorts, vorderhand, zudem, zunichte

(Präpositionen:) anhand, anstelle, aufgrund, infolge, mithilfe, zufolge, zugunsten

(Pronomen:) ebenderselbe, garnichts, irgendjemand, nirgendwo

R 19 Aus Fügungen entstandene Konjunktionen werden zusammengeschrieben.

B: inwiefern, obzwar, sobald, soda $\beta$, umso, wieweit, wohingegen 
R 20 Mehrteilige Numeralien werden zusammengeschrieben.

B: vierundzwanzig, siebenhundert[und]zweiunddreißig, neunzehnhundertsiebenundachtzig, sechsmillionendreihunderttausend;

acht[und]einhalb, zwei[und]dreiviertel;

der einhunderttausendste Teilnehmer; am vierundzwanzigsten Tage

\section{Substantiv}

R 21 Mehrteilige Substantive / Substantivierungen werden zusammengeschrieben. (Vgl. auch Bindestrichschreibung.)

B: Feuerstein, Kirschbaum, Kohlenwasserstoff, Lebenswerk, Sauerstoffflasche; Achterbahn. Fünfkampf; Altmeister, Faultier; Rastplatz, Stehpult; Außenpolitik, Nichtraucher; Selbstsucht;

das Autofahren, beim Spazierengehen, das Zustandekommen; das Allernötigste, die Leichtverwundeten, das Neugeborene; das Blaurot, das Schwarzrotgold;

das Stelldichein, das Vergißmeinnicht

5. Gemeinsamkeiten und Unterschiede in bezug auf die beschlossene Neuregelung

Der Vergleich des hier vorgestellten Regelungsvorschlages mit dem beschlossenen Regelwerk ergibt, daß zwischen ihnen einige wichtige Übereinstimmungen, aber auch gravierende Unterschiede bestehen. $\mathrm{Zu}$ den übereinstimmenden Kennzeichen gehören:

- das Bestreben, in dem als vereinfachungsbedürftig erkannten Bereich der GZS zu einer leichter handhabbaren, von Sonderfunktionen entlasteten Regelung zu kommen,

- die Nutzung der Signalfunktion der GZS zur Markierung nur des Unterschiedes zwischen Wortgruppe und Zusammensetzung und der Verzicht auf die unterscheidende Schreibung von konkretem vs. übertragenem Gebrauch bzw. von attributiver vs. prädikativer Verwendung,

- die Gliederung des Regelwerkes nach Wortarten,

- die Reduzierung der Regelzahl.

$\mathrm{Zu}$ den unterscheidenden Kennzeichen gehören vor allem die folgenden:

- Während bei der beschlossenen Neuregelung davon ausgegangen wird, daß die GS der Normalfall und daher allein die ZS regelungsbedürftig ist, werden im alternativen Vorschlag sowohl die ZS als auch die GS geregelt, wobei die Kriterien aus der für die einzelnen Wortarten spezifischen inhaltlichen Ausprägung des Grundunterschiedes zwischen Wortgruppe und Zusammensetzung bezogen werden.

- Während die Neuregelung auf Extensionalität, also auf Einbeziehung großer und zum Teil kompletter Beispiel-Fallgruppen in das Regelwerk setzt, zieht der alternative Vorschlag intensionale Regelformulierungen vor, die dem Schreibenden Kriterien für die Entscheidungsfindung gemäß seiner Aussageabsicht an die Hand geben. Die Beispiele dienen lediglich zur Illustration. 
- Während die Neuregelung als Folge ihres Hauptgrundsatzes, die GS als den Normalfall anzusehen, zu einer größeren Zahl von Schreibungsänderungen kommt, die - entgegen einer nachweislichen Entwicklungstendenz - bisherige Zusammenschreibungen zugunsten vermehrter GS wieder rückgängig machen, führt der alternative Vorschlag in Übereinstimmung mit der Sprachentwicklung zu leicht vermehrter ZS.

- Während die Neuregelung von Statik geprägt ist, versucht der alternative Vorschlag die für die GZS charakteristische Dynamik auch unter perspektivischem Aspekt dadurch zu berücksichtigen, daß die Regeln konsequent für die zum Zentrum des jeweiligen Kombinationstyps gehörenden Fälle formuliert werden. Der entwicklungsbedingte periphere Restbestand von nicht eindeutig zuordenbaren Fällen ist im Wörterverzeichnis nachzuschlagen. Dieses Verfahren soll es ermöglichen, sprachlichen Weiterentwicklungen, wie sie für die Peripherie typisch sind, im Wörterverzeichnis von Zeit zu Zeit durch Anpassungen der dort kodifizierten Schreibungsnorm zu entsprechen, ohne den Regelapparat als solchen verändern zu müssen.

Öhlschläger (1987: 33) scheint es fraglich, ob «die beiden skizzierten Vorschläge aber wirklich zu einer deutlichen Verbesserung der Regelung in diesem Bereich führen würden». Was unseren alternativen Vorschlag betrifft, so hat sich das immerhin für einen Teilaspekt, nämlich in bezug auf seine möglichen Auswirkungen auf den schulischen Aneignungsprozeß und auf das Fehlerbild von Schülern als wahrscheinlich herausgestellt. Küttel hat entsprechende Untersuchungen auf der Basis der von Herberg (1981) veröffentlichten Fassung, die mit der hier vorgestellten substantiell übereinstimmt, angestellt und kommt zu der generellen Bewertung, daß der Vorschlag «eine Erleichterung der GZS bewirken kann» (1983: 189).

\section{Literatur}

Duden (1985): Der Große Duden. Wörterhuch und Leitfaden der deutschen Rechtschreibung. 18. Neubearbeitung, Leipzig.

Herberg. Dieter (1975): Die geltende Regelung der Getrennt-und Zusammenschreibung und Ansatzpunkte zu ihrer Vereinfachung. In: Sprachwissenschaftliche Probleme einer Reform der deutschen Orhographie (I). Berlin, 88-122. (= Linguistische Studien, Reihe A/23)

Herberg, Dieter (1980): Zur Annahme eines lexikalischen Prinzips der Schreibung des Deutschen. In: Zeitschrift für Phonetik, Sprachwissenschaft und Kommunikationsforschung, 1, 3441.

Herberg, Dieier (1981): Untersuchungen zu einer Reform der deutschen Orthographie auf dem Gebiet der Getrennt-und Zusammenschreibung (GZS). In: Sprachwissenschaftliche Untersuchungen zu einer Reform der deutschen Orthographie (2). Berlin, 109-215 (= Linguistische Studien, Reihe A/83)

Herberg, Dieter (1986): Untersuchungen zur Getrennt- und Zusammenschreibung (GZS) in der deutschen Gegenwartssprache. Diss. Phil. B, Berlin.

Herberg, Dieter (1988): Getrennt- und Zusammenschreibung. Funktion, Regelung und Probleme. In: Deutschunterricht, 10, 465-472.

Küttel, Harmut (1983): Untersuchungen zur Gestaltung des Aneignungsprozesses bei der normrichtigen Getrennt- und Zusammenschreibung in der Disziplin Grammatik des Muttersprachunterrichts. Diss. Phil. B. Leipzig. 
Mentrup, Wolfgang (1986): Bericht über die internationale Arbeitstagung "Probleme der Rechtschreibung und ihre Neuregelung»: Zeichensetzung und Zusammen- und Getrenntschreibung (Mannheim, 2.-8. Juni 1986, Institut für deutsche Sprache). In: Deutsche Sprache, 3, 283-287.

Mentrup, Wolfgang (1987): Bericht über die 5. internationale Arbeitstagung "Probleme der Rechtschreibung und ihre Neuregelung»: Zeichensetzung. Getrennt- und Zusammenschreibung, Fremdwortschreibung (Zürich, 21.-26. September 1987). In: Deutsche Sprache, 4, 378-382.

Nerius, Dieter et al. (1989): Deutsche Orthographie. 2., durchgesehene Auflage, Leipzig.

Öhlschläger, Günther (1987): Möglichkeiten einer Reform der Getrennt- und Zusammenschreibung im Deutschen. In: GAL-Bulletin, 7, 30-35.

Schaeder, Burkhard (1989): «Probleme der deutschen Rechtschreibung und ihre Neuregelung»: Getrennt- und Zusammenschreibung, Schreibung mit Bindestrich, Fremdwortschreibung, Phonem-Graphem-Beziehung. Ein Bericht über die VI. internationale Arbeitstagung (Rostock, 10.-15. Oktober 1988). In: Deutsche Sprache, 1, 87-94. 